\title{
Telehealth Services for Home-based Rehabilitation of Cardiac Patients
}

\author{
Dieter Hayn ${ }^{1,2}$, Mahdi Sareban ${ }^{2,3}$, Alphons Eggerth ${ }^{1}$, Markus Falgenhauer ${ }^{1}$, Angelika Rzepka ${ }^{1}$, \\ Heimo Traninger ${ }^{4}$, Karl Mayr ${ }^{5}$, Marco Philippi ${ }^{6}$, Michael Porodko ${ }^{7}$, Christoph Puelacher ${ }^{8}$, Stefan \\ Höfer ${ }^{9}$, Josef Niebauer ${ }^{2,3}$ \\ ${ }^{1}$ AIT Austrian Institute of Technology, Graz, Austria \\ ${ }^{2}$ Ludwig Boltzmann Institute for Digital Health and Prevention, Salzburg, Austria \\ ${ }^{3}$ Universitätsinstitut für präventive und rehabilitative Sportmedizin, Paracelsus Medical University, \\ Salzburg, Austria \\ ${ }^{4}$ ZARG Zentrum für ambulante Rehabilitation GmbH, Graz, Austria \\ ${ }^{5}$ CARDIOMED Ambulantes kardiologisches Rehabilitationszentrum GmbH, Linz, Austria \\ ${ }^{6}$ MedReha GmbH, Feldkirch, Austria \\ ${ }^{7}$ Klinikum Wels-Grieskirchen, Wels, Austria \\ ${ }^{8}$ REHAMED-Tirol GmbH, Innsbruck, Austria \\ ${ }^{9}$ Medical University Innsbruck, Innsbruck, Austria
}

\begin{abstract}
Cardiovascular diseases (CVD) are the leading cause of death in the Western world. Several modifiable risk factors contribute to the pathogenesis of CVD which are all addressed during cardiac rehabilitation (CR). $C R$ is conducted in three phases: I: acute care hospital, II: subsequent in- or outpatient $C R$, and III: out-patient $C R$ with focus on lifelong prevention. Despite its proven merits, the adherence to healthy lifestyle changes following completion of CR phase II is challenging. This gap is addressed in recent recommendations, suggesting that clinicians should help patients to set personal goals to i) achieve and maintain the benefits of physical activity, ii) include physical activity into their daily routine and iii) overcome barriers to exercise, to achieve behavior change more effectively and more sustainably. We have developed tele-rehabilitation services to support patients during home-based exercise training in CR phase III. Our services provide a link between $C R$ experts and patients by means of individualized exercise prescription supported by different kinds of wearables for measuring e.g. physical activity volume. The effectiveness of such services and other supportive measures regarding adherence to home training plans and changes in exercise capacity during $C R$ phase III CR is currently evaluated in a study.
\end{abstract}

\section{Introduction}

Cardiovascular diseases (CVD) are the leading cause of death in the Western world with substantial micro- as well as macro-economic burden [1-3]. Several modifiable risk factors contribute to the pathogenesis of CVD which can be addressed during cardiac rehabilitation (CR). CR has proven to reduce mortality in CVD patients [4] and economic burden [5]. Increasing physical activity and exercise capacity are one of the main goals during CR because they have favorable effects on cardiovascular risk factors [6] and have shown to be strongly associated with lower mortality in CVD patients [7, 8].

CR is conducted in three phases: I: acute care hospital, II: subsequent in-clinic or out-patient CR, and III: outpatient $\mathrm{CR}$ with focus on lifelong prevention. Enrollment in CR phase II should take place as soon as possible once a patient meets one of the well-established indications [9]. CR phase II can be carried out in an inpatient (IN-II) or outpatient (OUT-II) CR facility. Notably, in the Austrian health care system, IN-II is standard, which stands in contrast to other European countries [10].

Following phase II, an outpatient phase III (OUT-III) enrollment is offered with weekly visits at the outpatient CR facility. In general, international secondary prevention guidelines advocate physical activity recommendations, i.e. $\geq 150 \mathrm{~min}$ of moderate intensity or $\geq 60$ to $75 \mathrm{~min}$ of vigorous exercise each week [11]. But it is well established that additional benefits without adverse effects occur with even more physical activity [12] and thus should be personalized and if indicated, gradually increased. Notably, the greatest benefits to health are likely to be achieved by increases in physical activity in subjects with more advanced disease and sedentary persons [7].

Despite its proven merits, the adherence to regular physical activity following completion of CR phase II seems challenging and the long-term preservation of recommended physical activity volume and exercise capacity is disappointing $[13,14]$. This gap is addressed in recent recommendations [11], suggesting that clinicians 
should help patients to set personal goals to i) achieve and maintain the benefits of physical activity, ii) include physical activity into their daily routine and iii) overcome barriers to exercise to achieve behavior change more effectively and more sustainably.

The rapid growth and availability of mobile health (mHealth) technology, such as wearable-trackers and smartphone-based application programs (mobile apps), provide a novel opportunity to address these recommendations. Notably, the proliferation of mobile technology reached even individuals 65 years or older with already four-in-ten seniors being smartphone owners and continuously increasing availability in these individuals. Different aspects of behavioral change, which pave the road to increase physical activity and exercise capacity, may be addressed by mHealth interventions: patient education, modelling, environmental restructuring and enablement as well as incentivisation and coercion [15, 16]. Additional opportunities of mHealth in $\mathrm{CR}$ are tailoring care to individual requirements and to get access to groups which are otherwise hard to reach.

The present paper describes the implementation and evaluation of telehealth services which support homebased CR, with the aim to increase long-term adherence to physical activity and improve the outcome of CR.

\section{Methods}

\subsection{Telehealth Services}

Two tele-rehabilitation services to support patients during home-based exercise training in CR phase III were developed. The services provide a link between CR experts and patients by means of individualized exercise prescription supported by different kinds of wearables for measuring e.g. physical activity volume. Details of both services are described in the following.

\section{2. heartfish app}

The heartfish app is a certified medical product designed to improve adherence to therapeutic and medical treatments in cardiometabolic prevention and rehabilitation. The core of the product are digital biomarkers, i.e. consumer-generated physiological and behavioral measures collected with digital tools. These data are used to continuously measure adherence to therapeutic and medical recommendations and to monitor the user's current cardiometabolic risk. Based on these digital biomarkers, the integrated dual coaching system enables service providers to intervene immediately and tailored to the individual needs of the patient. A screenshot of the app's activity screen is shown in Fig. 1.

\footnotetext{
${ }^{1}$ https://www.djangoproject.com/
}

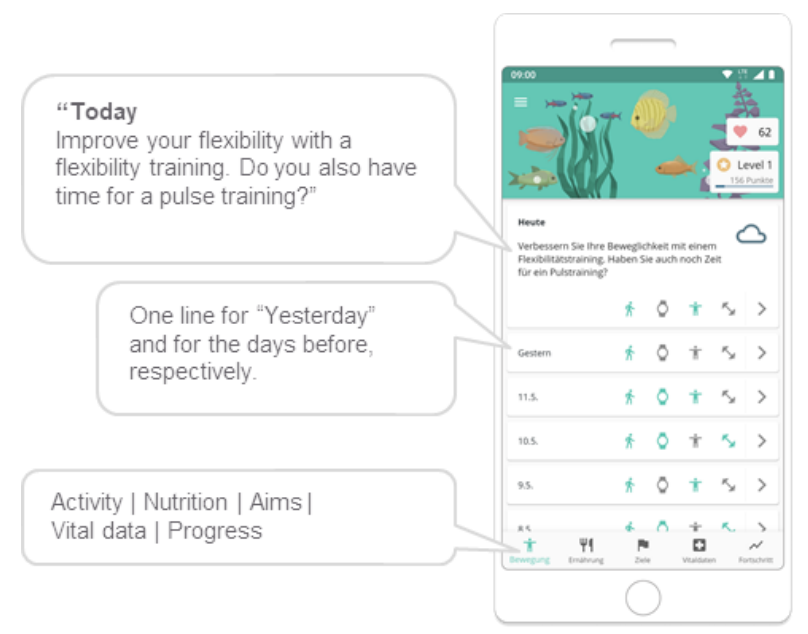

Figure 1. Screenshot of the heartfish app's activity screen with translations of the German texts (left)

\subsection{KIT telehealth service}

The KIT telehealth service is a certified medical product that consists of a backend service, which is based on the web framework django ${ }^{1}$ and programmed in Python, and a smartphone app running on Android.

The platform consists of specific application components (disease plugins) as well as a configurable core (core modules) and telemonitoring components (care module). The specific disease plugins provide optimum support for work procedures and functions relating to specific medical conditions. An overview of the components is shown in Fig. 2.

In the healthcare sector in Austria as well as other countries it is already possible, or will be in the foreseeable future, for health data to be shared electronically between institutions. Standardized document profiles in line with the HL7 standard for clinical document architecture (CDA) are used to integrate telemonitoring data seamlessly. Further simplification of telemedical support is anticipated, especially with the launch of the eMedication program.

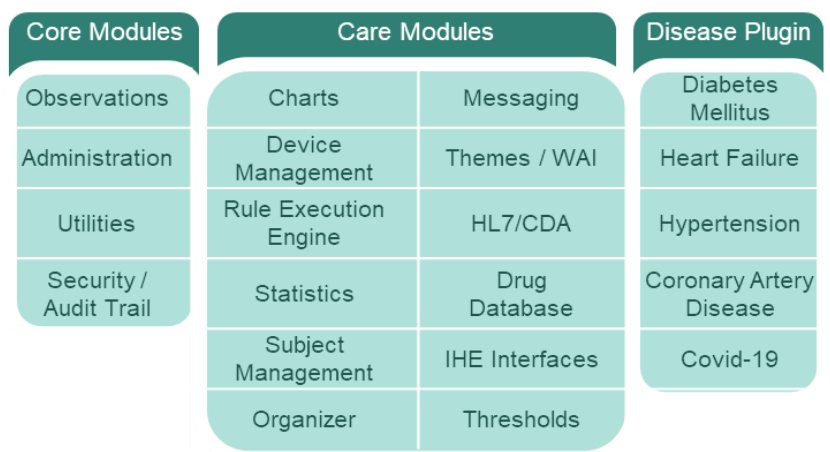

Figure 2. Components of the KIT telehealth service. 


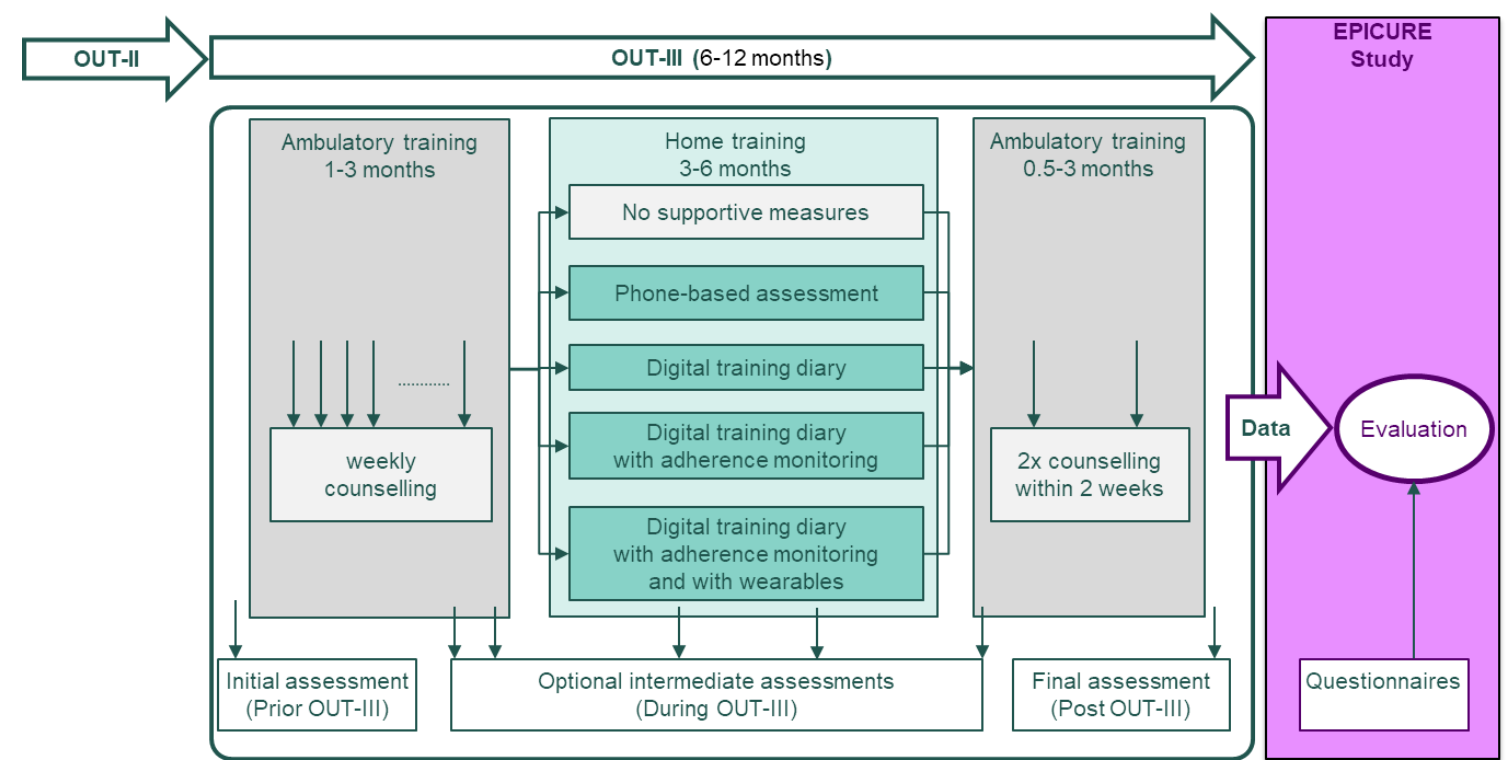

Figure 3 - Overview of cardiac rehabilitation phase III and EPICURE study.

\subsection{Evaluation}

The effectiveness of such services and other supportive measures regarding adherence to home training plans and changes in exercise capacity during $\mathrm{CR}$ phase III is currently evaluated in the EPICURE (Evaluation of multimodal supportive actions in outpatient cardiac rehabilitation in subjects with cardiovascular disease) study. The study protocol was approved by a local ethics committee and registered at ClinicalTrials.gov (NCT04458727). An overview of cardiac rehabilitation phase III and the EPICURE study is provided in Fig. 3.

\section{Results}

\section{1. heartfish app}

The medical concept on which heartfish is based reflects the requirements for outpatient CR and fits seamlessly into existing structures and processes. heartfish offers a customizable training program, individual recommendations, progress visualization, a multi-level adherence warning system and a dual coaching system to support both patients and service providers, especially during home-based training phases.

\subsection{KIT telehealth service}

For tele-rehabilitation, a specific disease plugin was developed. In addition to recording of various vital parameters (blood pressure, heart rate, body weight, subjective wellbeing, etc.), this plugin also supported a) tele-training with different kinds of activity trackers and smart watches and b) different training profiles (e.g. interval training, etc.).

\subsection{EPICURE study}

The EPICURE study started in autumn 2019 in five CR centers in Austria. At the moment (August 2020), 48 patients have finished the trial.

Out of five rehabilitation centers participating in the study, two centers used the heartfish app, two centers used the KIT telehealth service, and one center did not use a specific telehealth service to support their patient during home-based training.

Since - due to the CoVID-19 lockdown - recruitment was delayed especially in March to June 2020, we are currently prolonging the study period.

\section{Discussion}

Since the EPICURE study is still ongoing, details concerning the study results can only be analyzed and published after the end of the study.

During the CoVID-19 lockdown from March until June 2020, no CV at rehabilitation centers was allowed. However, home training as supported by our solution was possible. Therefore, patients already included in the study before the lockdown could continue their rehabilitation. Still, since no new patients could be recruited during the lockdown, the overall study duration had to be prolonged. However, the past months have shown that telehealth solutions might be a valuable alternative to training in the rehabilitation centers, especially while CoVID-19 is not completely under control.

\section{Conclusion}

Telehealth services for home-based rehabilitation of cardiac patients have the potential to improve long-term 
adherence to physical activity, even in situations when no training in rehabilitation centers is possible.

\section{Acknowledgments}

This project was funded by the Austrian Research Promotion Agency (FFG) within the COIN networking program, FFG No 865531.

\section{References}

[1] L. Jaspers, V. Colpani, L. Chaker, S. J. van der Lee, T. Muka, D. Imo, S. Mendis, R. Chowdhury, W. M. Bramer, A. Falla, R. Pazoki, and O. H. Franco, "The global impact of noncommunicable diseases on households and impoverishment: a systematic review," Eur J Epidemiol, vol. 30, no. 3, pp. 16388, Mar, 2015.

[2] G. N. D. C. Group, "Global, regional, and national burden of neurological disorders during 1990-2015: a systematic analysis for the Global Burden of Disease Study 2015," Lancet Neurol, vol. 16, no. 11, pp. 877-897, 11, 2017.

[3] P. A. Heidenreich, J. G. Trogdon, O. A. Khavjou, J. Butler, K. Dracup, M. D. Ezekowitz, E. A. Finkelstein, Y. Hong, S. C. Johnston, A. Khera, D. M. Lloyd-Jones, S. A. Nelson, G. Nichol, D. Orenstein, P. W. Wilson, Y. J. Woo, A. H. A. A. C. Committee, S. Council, C. o. C. R. a. Intervention, C. o. C. Cardiology, C. o. E. a. Prevention, C. o. Arteriosclerosis, T. a. V. Biology, C. o. Cardiopulmonary, C. Care, P. a. Resuscitation, C. o. C. Nursing, C. o. t. K. i. C. Disease, and a. I. C. o. Q. o. C. a. O. R. Council on Cardiovascular Surgery and Anesthesia, "Forecasting the future of cardiovascular disease in the United States: a policy statement from the American Heart Association," Circulation, vol. 123, no. 8, pp. 933-44, Mar, 2011.

[4] L. Anderson, N. Oldridge, D. R. Thompson, A. D. Zwisler, K. Rees, N. Martin, and R. S. Taylor, "Exercise-based cardiac rehabilitation for coronary heart disease cochrane systematic review and meta-analysis," Journal of the American College of Cardiology, vol. 67, no. 1, pp. 1-12, Jan, 2016.

[5] K. Edwards, N. Jones, J. Newton, C. Foster, A. Judge, K. Jackson, N. K. Arden, and R. Pinedo-Villanueva, "The costeffectiveness of exercise-based cardiac rehabilitation: a systematic review of the characteristics and methodological quality of published literature," Health Econ Rev, vol. 7, no. 1, pp. 37, Oct, 2017.

[6] J. Niebauer, K. Mayr, H. Harpf, P. Hofmann, E. Müller, M. Wonisch, R. Pokan, and W. Benzer, "Long-term effects of outpatient cardiac rehabilitation in Austria: a nationwide registry," Wien Klin Wochenschr, vol. 126, no. 5-6, pp. 148-55, Mar, 2014.

[7] R. A. H. Stewart, C. Held, N. Hadziosmanovic, P. W. Armstrong, C. P. Cannon, C. B. Granger, E. Hagström, J. S. Hochman, W. Koenig, E. Lonn, J. C. Nicolau, P. G. Steg, O. Vedin, L. Wallentin, H. D. White, and S. Investigators, "Physical activity and mortality in patients with stable coronary heart disease," J Am Coll Cardiol, vol. 70, no. 14, pp. 16891700, Oct, 2017.

[8] S. A. Lear, W. Hu, S. Rangarajan, D. Gasevic, D. Leong, R. Iqbal, A. Casanova, S. Swaminathan, R. M. Anjana, R. Kumar, A. Rosengren, L. Wei, W. Yang, W. Chuangshi, L. Huaxing, S.
Nair, R. Diaz, H. Swidon, R. Gupta, N. Mohammadifard, P. Lopez-Jaramillo, A. Oguz, K. Zatonska, P. Seron, A. Avezum, P. Poirier, K. Teo, and S. Yusuf, "The effect of physical activity on mortality and cardiovascular disease in 130000 people from 17 high-income, middle-income, and low-income countries: the PURE study," Lancet, vol. 390, no. 10113, pp. 2643-2654, Dec, 2017.

[9] J. Niebauer, K. Mayr, M. Tschentscher, R. Pokan, and W. Benzer, "Outpatient cardiac rehabilitation: the Austrian model," Eur J Prev Cardiol, vol. 20, no. 3, pp. 468-79, Jun, 2013.

[10] B. Bjarnason-Wehrens, H. McGee, A. D. Zwisler, M. F. Piepoli, W. Benzer, J. P. Schmid, P. Dendale, N. G. Pogosova, D. Zdrenghea, J. Niebauer, M. Mendes, and C. R. S. E. A. o. C. P. a. Rehabilitation, "Cardiac rehabilitation in Europe: results from the European Cardiac Rehabilitation Inventory Survey," Eur J Cardiovasc Prev Rehabil, vol. 17, no. 4, pp. 410-8, Aug, 2010.

[11] M. F. Piepoli, A. W. Hoes, S. Agewall, C. Albus, C. Brotons, A. L. Catapano, M. T. Cooney, U. Corrà, B. Cosyns, C. Deaton, I. Graham, M. S. Hall, F. D. R. Hobbs, M. L. Løchen, H. Löllgen, P. Marques-Vidal, J. Perk, E. Prescott, J. Redon, D. J. Richter, N. Sattar, Y. Smulders, M. Tiberi, H. B. van der Worp, I. van Dis, W. M. M. Verschuren, S. Binno, and E. S. D. Group, "2016 European Guidelines on cardiovascular disease prevention in clinical practice: The Sixth Joint Task Force of the European Society of Cardiology and Other Societies on Cardiovascular Disease Prevention in Clinical Practice (constituted by representatives of 10 societies and by invited experts)Developed with the special contribution of the European Association for Cardiovascular Prevention \& Rehabilitation (EACPR)," Eur Heart J, vol. 37, no. 29, pp. 2315-2381, 08, 2016.

[12] J. Sattelmair, J. Pertman, E. L. Ding, H. W. Kohl, W. Haskell, and I. M. Lee, "Dose response between physical activity and risk of coronary heart disease: a meta-analysis," Circulation, vol. 124, no. 7, pp. 789-95, Aug, 2011.

[13] S. A. Lear, J. J. Spinelli, W. Linden, A. Brozic, M. Kiess, J. J. Frohlich, and A. Ignaszewski, "The Extensive Lifestyle Management Intervention (ELMI) after cardiac rehabilitation: a 4-year randomized controlled trial," Am Heart J, vol. 152, no. 2, pp. 333-9, Aug, 2006.

[14] C. M. Yu, L. S. Li, H. H. Ho, and C. P. Lau, "Long-term changes in exercise capacity, quality of life, body anthropometry, and lipid profiles after a cardiac rehabilitation program in obese patients with coronary heart disease," Am J Cardiol, vol. 91, no. 3, pp. 321-5, Feb, 2003.

[15] L. Neubeck, N. Lowres, E. J. Benjamin, S. B. Freedman, G. Coorey, and J. Redfern, "The mobile revolution--using smartphone apps to prevent cardiovascular disease," Nat Rev Cardiol, vol. 12, no. 6, pp. 350-60, Jun, 2015.

[16] S. Michie, M. M. van Stralen, and R. West, "The behaviour change wheel: a new method for characterising and designing behaviour change interventions," Implement Sci, vol. 6, pp. 42, Apr, 2011.

Address for correspondence:

Dieter Hayn

Reininghausstraße 13/1, 8020 Graz, Austria

dieter.hayn@ait.ac.at 